\title{
Cell therapy for cartilage defects of the hip
}

\author{
Rodrigo Mardones ${ }^{1,2}$ \\ Alessio Giai Via ${ }^{1}$ \\ Claudio Jofré ${ }^{2}$ \\ José Minguell2 \\ Claudio Rodriguez ${ }^{1}$ \\ Alexander Tomic ${ }^{1}$ \\ Matias Salineros ${ }^{1}$ \\ 1 Department of Orthopaedic Surgery, Adult Recon- \\ struction Surgery Hip/Knee and Hip Arthroscopy, \\ Santiago de Chile, Chile \\ 2 Regenerative Cell Therapy Center, Clínica Las \\ Condes, Santiago de Chile, Chile
}

Corresponding author:

Rodrigo Mardones, MD

Department of Orthopaedic Surgery, Adult Reconstruction Surgery Hip/Knee and Hip Arthroscopy, La Fontecilla 441, Las Condes, Santiago de Chile, Chile E-mail: rmardones@clc.cl

\section{Summary}

Background: Chondral injuries are commonly related to poor clinical outcome, but recent data showed some improvements in function and pain after hip arthroscopy. Cell-based therapies represent an appealing alternative strategy for cartilage regeneration, and interesting results have been recently reported after intra-articular injections of mesenchymal stem cells (MSCs). The results of hip arthroscopy for femoroacetabular impingement (FAI) and intra-articular injections of autologous expanded bone marrow - MSCs (BMMSCs) are reported in this retrospective study.

Materials and methods: Twenty patients (29 hips) received hip arthroscopy for FAl and focal cartilage injuries or mild to moderate osteoarthrosis (OA). Three intra-articular injections of $20 \times 10^{6}$ BM-MSCs were injected from 4 to 6 weeks postoperative. The modified Harris Hip score (mHHS), the WOMAC score, the VAIL score and VAS score were administered to all patients.

Results: The mean age of the patients was $\mathbf{5 1 . 8}$ years, and the mean follow-up was 24 months. The median preoperative mHHS, WOMAC and VAIL scores were $64.3,73$ and 56.5 respectively, and they increased to 91,97 and 83 at final follow up $(p<0.05)$. The VAS score also improved from a median of 6 to 2 . Four patients received a THA (13\% of the hips) at the median of 9 months post intervention (range 6-36 months). Six patients referred pain after the injection of MSCs, which improved with oral pain killers. No major complications were reported.

Conclusion: BM-MSCc injections in combination with hip arthroscopy may improve the quality of life and functional score in patient with FAI and cartilage injuries which are still not candidate to a THA. Level of evidence: IV case series.

KEY WORDS: cartilage injuries, femoroacetabular impingement, hip arthroscopy, mesenchymal stem cells, osteoarthrosis, stem cells therapy.

\section{Introduction}

Cartilage injuries of the hip are commonly related to relatively poor clinical outcome after hip arthroscopy, and severe osteoarthrosis (OA) is considered a contraindication by many Authors. However, recent data showed that femoroacetabular impingement (FAI) correction with mild to moderate articular cartilage degeneration resulted in improvement of pain and function at short-term follow-up ${ }^{1,2}$. Treatment of articular cartilage defects is challenging, and a gold standard therapy is not available yet. Cell-based therapies using either chondrocytes or mesenchymal stem cells (MSCs) represent an appealing alternative strategy. Different protocols of intra-articular MSCs injection have been studied to treat chondral injuries in large animals such as sheep, pigs and horses $^{3}$. Even if the results are still conflicting, some studies showed that intra-articular injections of MSCs are able to produce a good defect coverage with hyaline-like cartilage ${ }^{4}$. According to these interesting preclinical results, support for MSCs-based therapies in clinical practice have been provided ${ }^{3}$. Recently, some Authors reported on intra-articular injection of MSCs into the knee for the treatment of focal defect or more generalized cartilage loss in osteoarthritis, showing interesting results regard pain and clinical outcome $e^{5,6}$. Furthermore, no serious adverse effects to the application of expanded MSCs have been reported to date, beyond transient pain and inflammationn ${ }^{3}$. Even if the currents knowledge about intra-articular MSCs injection is based only on knee studies, hip 
arthroscopy followed by autologous MSCs infusion for patients with large chondral defect or mild-moderate OA may produces better outcome. The aim of this study is to evaluate the functional outcome and postoperative complications of patients undergoing hip arthroscopy for FAI and intra-articular infiltrations of autologous expanded bone marrow-MSCs (BMMSCs) at mid-term follow-up.

\section{Materials and methods}

We reviewed retrospectively the data of sixty-three patients who received hip arthroscopy and intra-articular BM-MSCs injections, from 2012 to 2015, for FAI and large focal chondral delaminations (Outherbridge Grade III-IV) or mild to moderate OA. Both surgical and study procedures were performed after the patients had signed a written consent and after approval by the local Internal Review Board (IRB-Clínica Las Condes, Santiago de Chile, Chile). This research has been conducted ethically according to international standards as required by the journal ${ }^{7}$.

\section{Inclusion and exclusion criteria}

The inclusion criteria were patients with symptomatic FAI and focal grade III-IV chondral defects according Outherbridge classification, or mild to moderate OA (Tönnis scale II-III). Patients older than 18 and younger than 60 years were included into the study. The exclusion criteria were OA secondary to hip developmental diseases (developmental hip dysplasia, Perthes disease, slipped capital femoral epiphysis), previous intra-articular fractures, autoimmune OA, previous joint infections, and patients with symptomatic lumbar spine disorders. Patients who left the follow protocol or with a follow-up shorter than one year have also been excluded.

\section{Patient characteristics and assessment}

Twenty patients met the inclusion criteria. Nine pa- tients were treated bilaterally, for a total of 29 hips. The mean age of the patients (10 women and 10 men) was 51.4 years (range 39-60; median 50; SD 6.4), and the mean follow-up was 24 months (range 13-36 months; SD 7). We excluded 11 patients from our cohort because 2 patients had a borderline DDH and 1 patient received a previous periacetabular osteotomy, 2 patients had a previous osteonecrosis of the femoral head, 1 patient was affected by a chronic cruralgia, and 5 patients were lost at follow-up.

All patients took standard weight-bearing antero-posterior (AP) X-ray of the pelvis, AP and cross-table view of both hips (Figure 1). Cam impingement was assessed by the measurement of the a-angle on the cross-table view of the hip and femoral head-neck offset $^{8}$. An alpha angle of greater than $50^{\circ}$ and a femoral head-neck offset lower than $10 \mathrm{~mm}$ were considered pathologic ${ }^{9}$. Pincer impingement was defined as a lateral center edge angle (LCEA) by Wiberg greater than $35^{\circ}$ and/or an extrusion index smaller than $20 \%{ }^{10}$. An artro-MRI of the affected hip was performed to diagnose the labral injury, chondral defects and other intra-articular pathologies (Figure 2). All patients underwent a diagnostic intra-articular lidocaine injection to confirm the diagnosis.

The modified Harris Hip Score (mHHS) and the Western Ontario and McMaster Universities Osteoarthritis Index (WOMAC) were administered to all patients before surgery (T0) and final follow-up (T1). The VAS score was also administered preoperatively and at each control. The radiological evaluation was performed with Kodak ${ }^{\circledR}$ Carestream PACS $\AA 2.0$ software. Evaluation of clinical data was performed from the electronic record of institutional patients with Power-Chartsoftware. A failure of a treatment failure was considered the need of Total Hip Arthroplasty (THA). Short term complication after the injection (infection/ pain/arthritis) were also evaluated.

\section{Surgical technique}

All hip arthroscopies were performed by the same op-

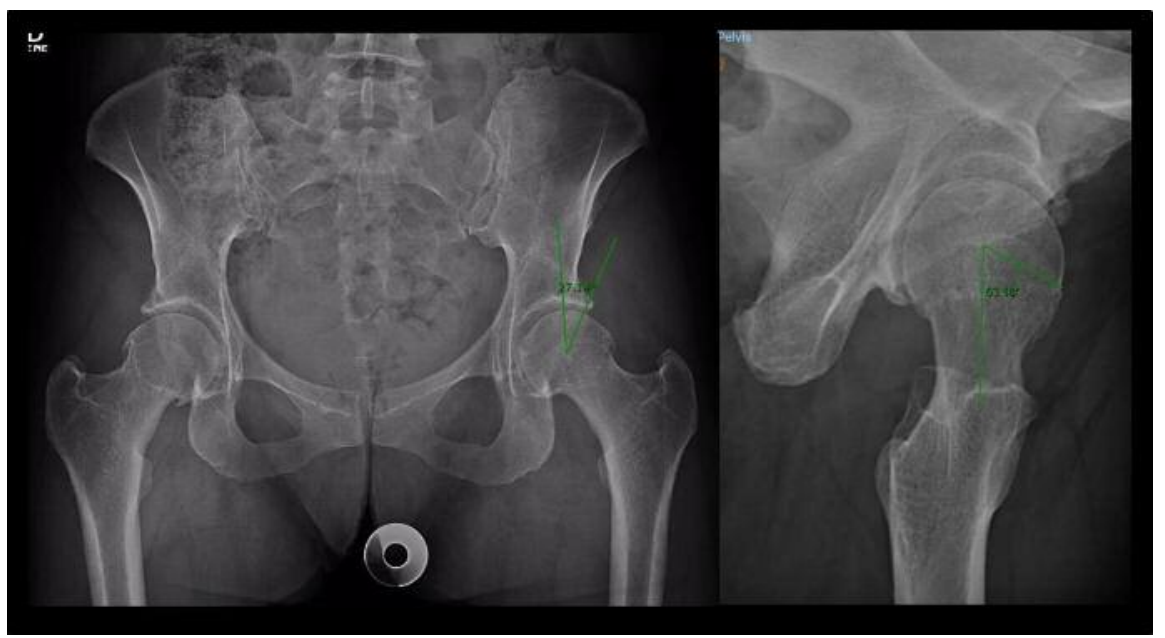

Figure 1. Pre-operative $X-$ ray showing femoroacetabular impingement CAM-Type (Wiber angle of $26^{\circ}$; a-angle of $63^{\circ}$ ) and osteoarthrosis of both hips. 


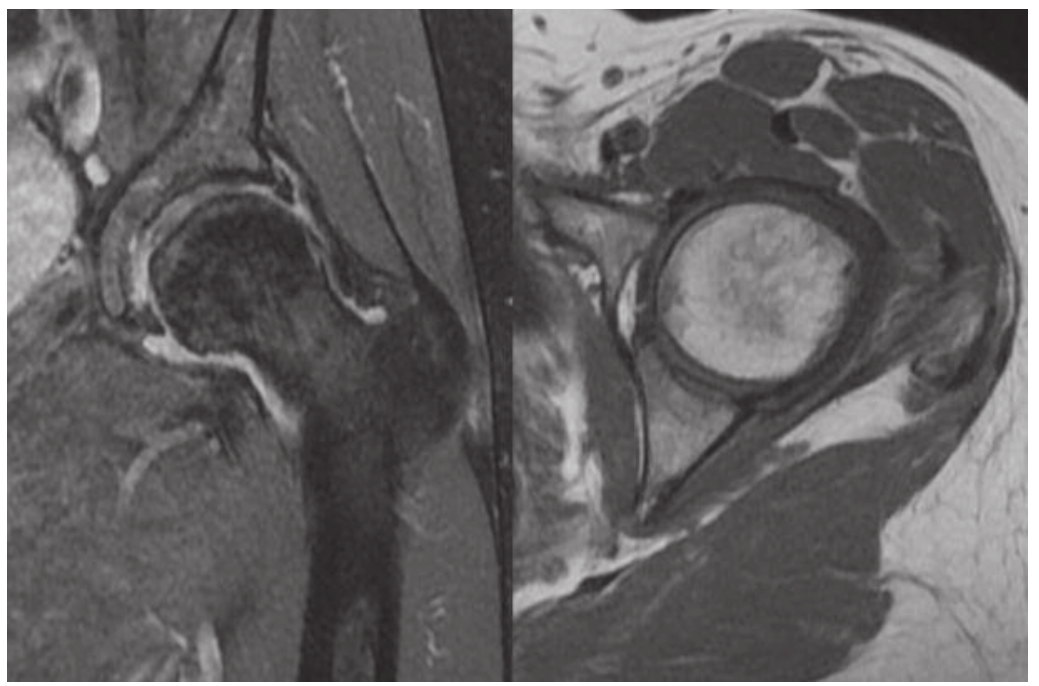

Figure 2. The artro-MRI of the left hip shows chondral injuries at the acetabular side.

erator (the signor Author RM). The patient was positioned supine on a fracture table, under combined anesthesia (spinal anesthesia and deep sedation). The affected hip was prepared and draped in the usual sterile fashion, and traction was applied to open the articular space. A standard anterolateral portal and a second modified anteromedial portal were used $^{11}$. The anterolateral portal was performed under radioscopy guidance, while the antero-medial portal under direct visualization. A $70^{\circ}$ arthroscope was used. The arthroscopy of the central compartment was performed first. Labral injuries, chondro-labral and chondral defects (Figure 3 ), the presence of a prominent antero-inferior iliac spine (AIIS) ${ }^{12}$, and ligamentum teres tendinopathy were detected. In case of focal or global acetabular overcorage, acetabuloplasty was performed, while when a prominent AIIS was observed (type II and III according Hetsroni) ${ }^{12}$ a partial resection of the spine was performed. Labral teres were repaired with 2 to 5 bonny anchors, and chondral defects were treated with microfractures. After performing the arthroscopy of the central compartment, traction was removed and the external compartment was approached. In case of CAM-impingment, the bump was removed and the resection was controlled with the aid of fluoroscopy.

\section{BM-MSCs harvest, expansion and injection}

Eighty $\mathrm{mL}$ of bone marrow were aspirated from the anterior iliac crest during hip arthroscopy. Mononuclear cells (MNC) were isolated by Lymphoprep ${ }^{\mathrm{TM}}$ density gradient (Figure 4) and the sample containing bone marrow derived-mononuclear cells (BM-MNCs) is seeded in $225 \mathrm{~cm}^{2}$ cell culture flasks at a cell density of 10.000 cells $/ \mathrm{cm}^{2}$. The primary cell culture reaches $70-80 \%$ confluence within $2-3$ weeks. Cell cultures is harvest using tripsin-EDTA $(0,05 \%)$ for 5 minutes, counted and cell viability by trypan blue assay in a

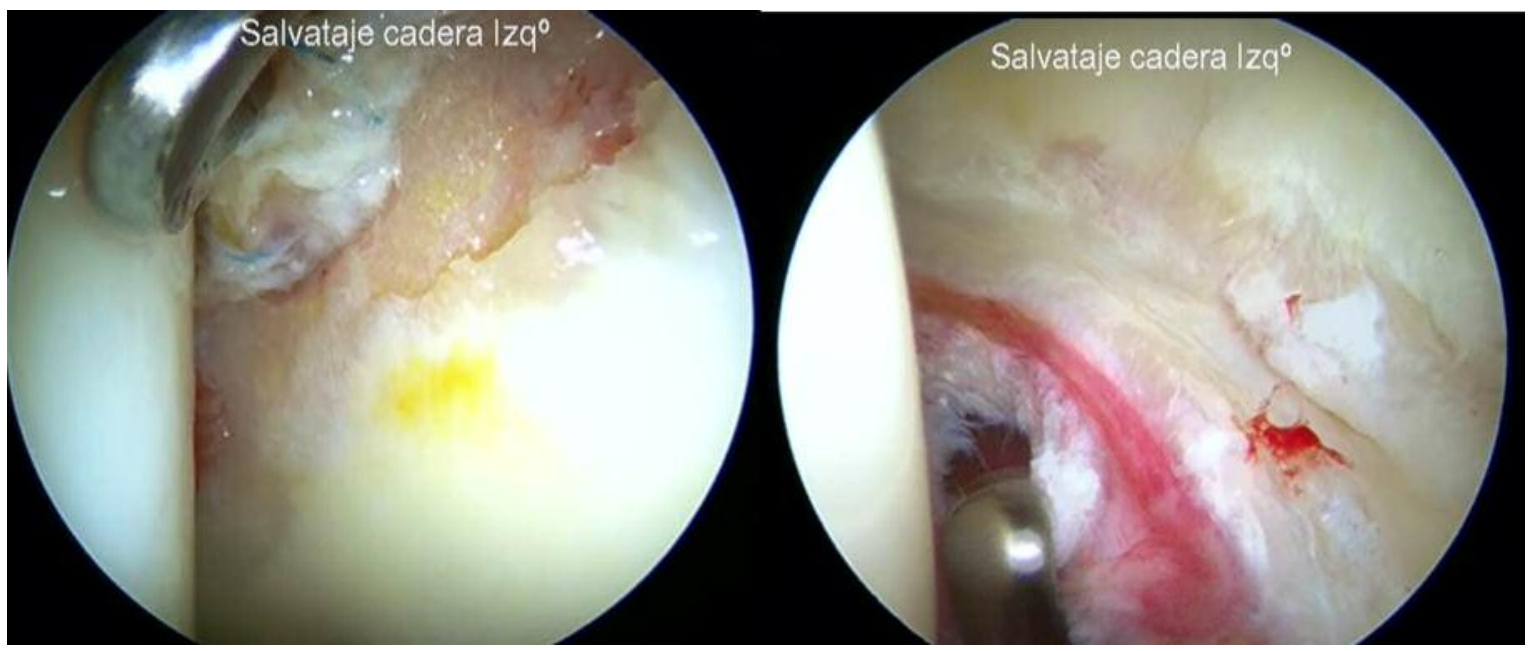

Figure 3. Intra-operative images: large grade III and IV chondral defects at the chondro-labral junction. 


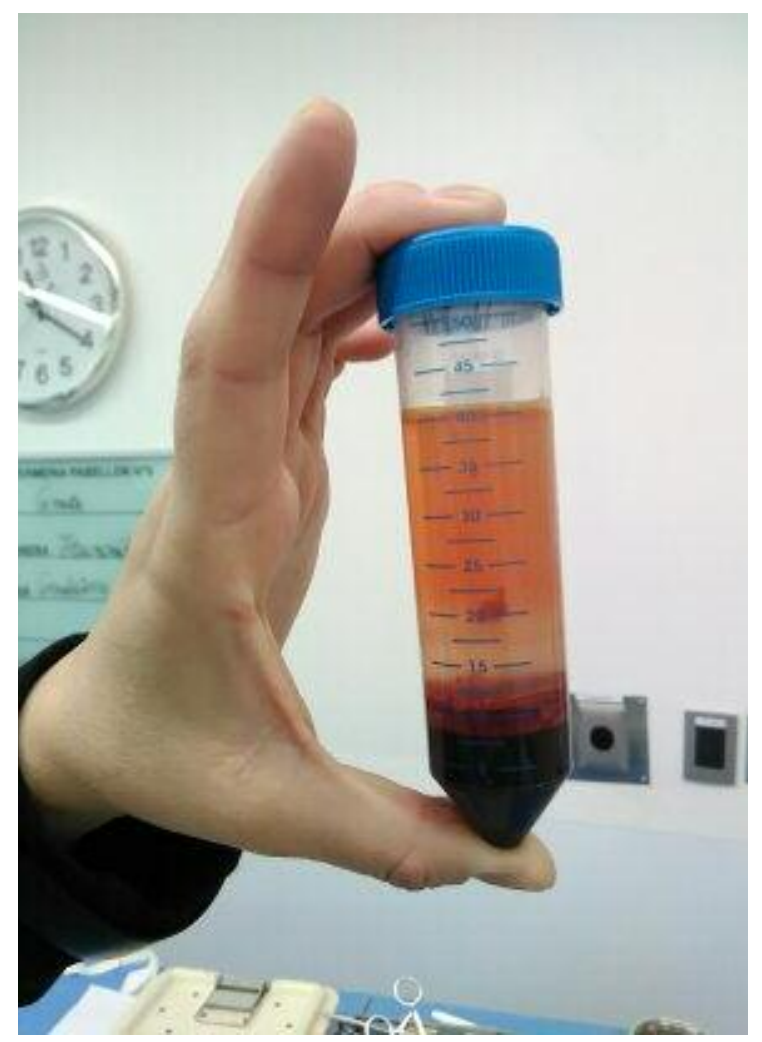

Figure 4. Bone marrow is aspirated from the anterior iliac crest just before the arthroscopic procedure, and the mononuclear cells are isolated by Lymphoprep ${ }^{\mathrm{TM}}$ density gradient.

automatic cell counter, EVE Cell Counter (Nanoentek $^{\mathrm{TM}}$ ) was performed.

When the cultures reach the required number $\left(20 \times 10^{6}\right.$ cells), several cell analyses are performed: endotoxin, micoplasm, and microbiological tests from all cell concentrates. Besides, immunotypification by flow cytometry panel for MSC markers CD73+,
CD90+, CD105+, CD34- and CD45- were evaluated. Finally, cell concentrates were resuspended in a physiological saline solution supplemented with $0,02 \%$ human albumin serum (Griffols ${ }^{\circledR}$ ) in a total volume of $1,5 \mathrm{~mL}$. Each patient received 3 intra-articular injections of $20 \times 10^{6}$ cells, one per week, 4 to 6 weeks post-operative. An expert radiologist performed the injections under radioscopic guidance.

\section{Postoperative protocol}

All patients underwent standard plain AP X-ray of the pelvis and cross-table radiographs of the hip while supine the day after surgery (Figure 5). The hip was mobilized immediately after surgery by a passive mobilizer (kinetic $\left.{ }^{\circledR}\right)$. Then, active mobilization with a static bicycle twice a day for 15 minutes was started in the first postoperative day, and walking with two crutches and weight bearing as tolerated was allowed. All patients received the same rehabilitation program by well-trained physiotherapist of our clinic.

\section{Statistical analysis}

The analytical research was performed with Microsoft Excel $2011{ }^{\circledR}$ software, and statistical analysis was performed with STATA $12,1 \AA$ and EPIDAT $4,1 \AA$ software. Descriptive statistics are presented as mean $( \pm S D)$. Student's t-test was used to detect for differences between baseline and follow-up for each variable. A p-value $<0.05$ was considered to be statistically significant.

\section{Results}

\section{Radiological findings}

The Tönnis grade evaluated on the AP X-ray of the pelvis was I in 7 patients, II in 17 hips, III in 5 hip. At the end of the follow-up, we found an evolution of the hip osteoarthrosis grade in 6 hips.

\section{Clinical and Functional Assessment}

The median preoperative mHHS was 64.3 (range, 45-

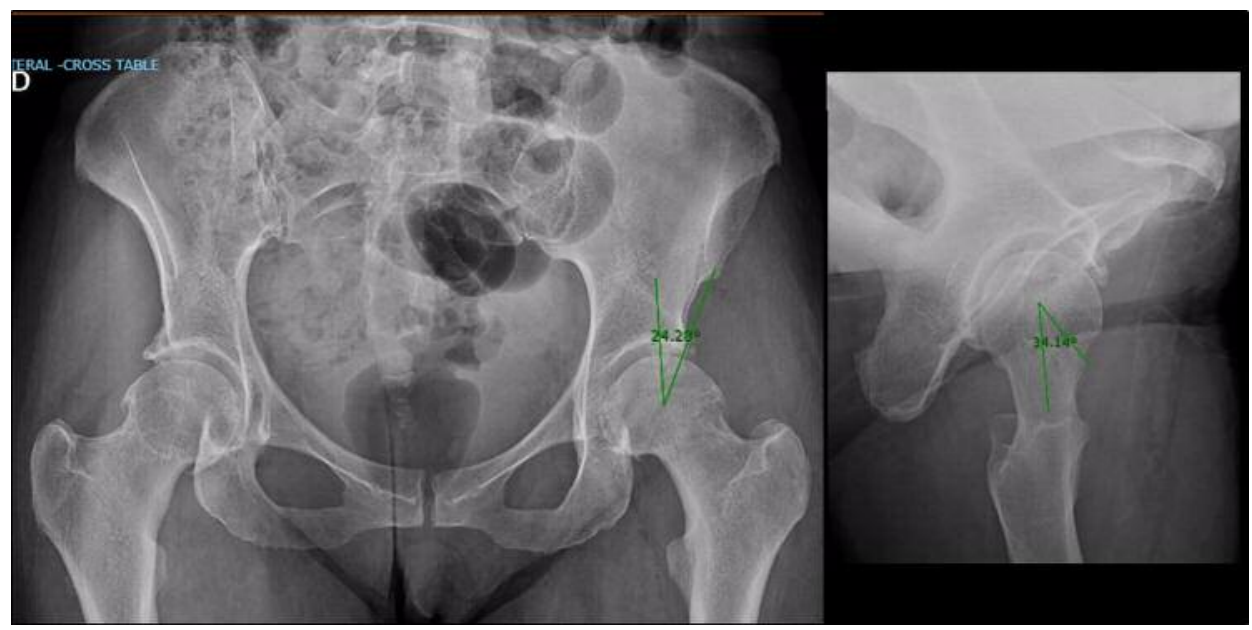

Figure 5. Postoperative X-ray. 
93; $\mathrm{SD}=14.3$ ) and increased to 91 (range, 70-100; $S D=11.2$ ) at final follow-up. The median WOMAC and VAIL scores increased from 74 (range, 23-95; $\mathrm{SD}=19.6$ ) and 56.6 (range, 31-97; $S D=16.2$ ) respectively to 97 (range, 64-84; SD=6.08) and 83 (range,56-100; $S D=15.3$ ) at final follow-up. The VAS score improved from a median of 6 preoperatively (range, 2-8; $S D=1.3$ ) to 2 (range, $0-5 ; S D=1.6$ ). All these results were statistically significant $(p<0.05)$. Four patients received a THA during the follow-up (13.8\% of the hips), at the median of 9 months post intervention (range 6-36 months). Two of these patients had a preoperatory Tönnis grade of II and two of III.

We found also an improvement of $10^{\circ}$ of internal rotation in most of patients, with the hip both at $0^{\circ}$ and $90^{\circ}$ of flexion, but the difference was not statistically significant.

\section{Complications}

No major complications nor infection were reported after hip arthroscopy and the intra-articular injections of MSCs. But 6 patients referred pain after the second or third injection of MSCs (VAS score range from 2 to 9 ), which improved with oral pain killers.

\section{Discussion}

The main finding of the present study is that hip arthroscopy and adjuvant intra-articular injections of expanded BM-MSCs seems to be safe and produce interesting results at middle-term follow-up in patients with large chondral defects or mild osteoarthrosis of the hip which are still not candidate or refuse a THA. Chondral injuries and $\mathrm{OA}$ are frequent joint disease, characterized by a progressive and irreversible degeneration of articular cartilage and impaired functions. Different therapies have been developed to treat and slow the articular degenerative changes, but a gold standard treatment is not available yet. MSCs are an attractive candidate for cartilage repair. MSCs can be harvested from various tissue (bone marrow, adipose tissue, umbilical cord blood, synovial membrane and others), they have a self-renewal capacity and a chondrogenic differentiation potential ${ }^{13}$. In addition, MSCs produces a variety of extracellular matrix macromolecules involved in cartilage function, including collagen, fibronectin, glycosylaminoglycans (GAGs) and proteoglycans, as well as many cytokines, growth factors, colony stimulating factors and chemokines. MSCs therapy may act via two ways, preventing cartilage degradation through the secretion of bioactive factors and anti-inflammatory cytokines by endocrine, paracrine and autocrine functions ${ }^{13-15}$, and by differentiating into chondrocytes and contributing to cartilage repair ${ }^{16}$. Secretion of bioactive mediators by MSCs may prevent loss of chondrocyte anabolic activity or stimulate progenitors present in the cartilage ${ }^{17,18}$. MSCs therapies showed interesting results in regenerating articular cartilage in several animal studies ${ }^{3}$, and intra-articular MSCs injections have been supported by recent literaturee ${ }^{19,20}$. Different MSCs injection protocols have been studied to treat large chondral defects in animals. Even if direct implant of MSCs seems to be more effective than intra-articular injections in covering focal defects with cartilage like tissue, Lee et al. ${ }^{4}$ reported good defect coverage with hyaline-like cartilage in an animal porcin model at 12 weeks after injection of MSCs and hyaluronic acid (HA). Integrated repair tissue consistent with hyaline cartilage was found in a simulated chondral defects in a simulated chondral defect of the distal femur of goats after MSCs injections at 6 months after treatment ${ }^{21}$. The injections were performed weekly for 3 weeks. In a similar model, Saw et al. ${ }^{22}$ used concentrated BMMSCs injected weekly with HA. They found improved content of proteoglycan and type II collagen within femoral trochlea defects that received cell injections in comparison to those that received only HA. Furthermore, the intra-articular injection may be useful in cases of diffuse OA, as showed by ter Huurne et al ${ }^{23}$. They reported that a single injection of AD-MSCs of mice with early-stage collagenase-induced $O A$ inhibits synovial thickening and cartilage destruction. Even if few clinical studies are currently available in literature about direct intra-articular injection of MSCs, and all data arise from knee models, encouraging results have been recently reported. Jo et al. ${ }^{24}$ reported the results of 18 patients with knee OA and intra-articular injection of AD-MSCs. Patients were divided into 3 groups who received a low-dose $(1 \mathrm{x}$ $\left.10^{7}\right)$, a mid-dose $\left(5 \times 10^{7}\right)$, and high-dose $\left(1 \times 10^{8}\right)$ AD-MSCs. The Authors found improvement in pain and function, and a significantly decreased of the size of cartilage defect at the MRI in the high-dose group at 6 months. The second-look arthroscopy and histological findings also showed a consistently decreased of articular cartilage defects by regeneration of hyaline-like articular cartilage. Furthermore, no major adverse effect have been reported. The results of 30 patients older than 65 years with knee OA have been recently published 25 . A mean of $4 \times 10^{7}$ AD-MSCs and PRP were injected into the knee after arthroscopic lavage. The Authors found good results in term of improved pain and function at 2 years follow-up. The second-look arthroscopy was performed in 16 patients and showed the improvement or maintained cartilage status in 14 of them. Five patients demonstrated a worsening of OA according the KellgrenLawrence scale, but no patients underwent total knee arthroplasty. Knee pain was reported during the first week after the stem cell injection which resolved with oral pain killers.

However, there are still many unanswered questions for MSCs therapy, such as, which is the most appropriate tissue source? Is the action of MSCs direct, or they act by an indirect (endocrine) mechanism? Which factors released by MSCs have a chondroprotective effect? Furthermore, there is still a controversy about the best dosage and processing methods ${ }^{14}$. In fact, clinical results seem to be positively related to the number of cells injected, but a standardization of 
MSCs dose is difficult due to a lack of uniformly between studies 26,27 .

We acknowledge of the limitations of the present study. One limitation of this study is a relatively short follow-up that cannot assess long-term results of intra-articular MSCs injections, which would determine whether there is some influence on the progression of the ostheoarthritic disease or is just a relieving of symptoms. The small sample size is a limitation which made statistical analysis of the data difficult. The absence of a control group does not allow to draw a clear superiority of MSCs injection compared to the other treatments. Thus, chondral injuries and hip OA are chronic diseases and long-term outcomes should be an important consideration in evaluating new treatments.

\section{Conclusion}

A gold standard procedure to treat large chondral defects of the hip is still lacking. In order to reduce the evolution of hip OA and improve symptoms, hip arthroscopy followed by adjuvant intra-articular injections of expanded BM-MSCs seems to produce interesting results. No major complications have been reported after treatment. However, further studies involving more patients and with larger follow-up are needed.

The Authors declare that this mini-review was conduct according ethically to international standards and as required by the journal as described.

\section{References}

1. Daivajna S, Bajwa A, Villar R. Outcome of Arthroscopy in Patients with Advanced Osteoarthritis of the Hip. PLoS ONE 2015;10:1-6.

2. Mardones R, Nemtala F, Tomic A. Arthroscopic Treatment of Femoroacetabular Impingement in Patients over 60 Years Old: Preliminary Report of a Pilot Study. Cartilage. 2010;1: 188-193.

3. Bornes TD, Adesida AB, Jomha NM. Mesenchymal stem cells in the treatment of traumatic articular cartilage defects: a comprehensive review. Arthritis Research \& Therapy. 2014; 16:432-451.

4. Lee KB, Hui JH, Song IC, Ardany L, Lee EH. Injectable mesenchymal stem cell therapy for large cartilage defects - a porcine model. Stem Cells. 2007;25:2964-2971.

5. Davatchi F, Abdollahi BS, Mohyeddin M, et al. Mesenchymal stem cell therapy for knee osteoarthritis. Preliminary report of four patients. Int J Rheum Dis. 2011;14:211-215.

6. Emadedin M, Aghdami N, Taghiyar L, et al. Intra-articular injection of autologous mesenchymal stem cells in six patients with knee osteoarthritis. Arch Iran Med. 2012;15: 422-428.

7. Padulo J, Oliva F, Frizziero A, Maffulli N. Muscles, Ligaments and Tendons Journal. Basic principles and recommendations in clinical and field science research: 2016 Update. MLTJ. 2016;6(1):1-5.

8. Cefin Barton C, Salineros MJ, Rakhra KS, Beaule PE. Validity of the Alpha Angle Measurement on Plain Radiographs in the
Evaluation of Cam-type Femoroacetabular Impingement. Clin Orthop Relat Res 2011;469:464-469.

9. Nemtala F, Mardones R, Tomic A. Anterior and Posterior Femoral Head-Neck Offset Ratio in the Cam Impingement. Cartilage 2010;1:238-241.

10. Tannast M, Siebenrock KA, Anderson SE. Femoroacetabular Impingement: Radiographic Diagnosis-What the Radiologist Should Know. AJR 2007; 188:1540-1552.

11. Byrd T. Modified Anterior Portal for Hip Arthroscopy. Arthr Tech 2013; 2:337-339.

12. Hetsroni I, Poultsides L, Bedi A, Larson CM, Kelly BT. Anterior Inferior lliac Spine Morphology Correlates With Hip Range of Motion: A Classification System and Dynamic Model. Clin Orthop Relat Res. 2013;471:2497-2503.

13. Via AG, Frizziero A, Oliva F. Biological properties of mesenchymal Stem Cells from different sources. Muscles Ligaments Tendons J. 2012 16;2:154-62.

14. Mardones R, Jofré CM2, Minguell JJ. Cell Therapy and Tissue Engineering Approaches for Cartilage Repair and/or Regeneration. Int J Stem Cells. 2015;8.

15. Filardo G, Madry H, Jelic M, Roffi A, Cucchiarini M, Kon E. Mesenchymal stem cells for the treatment of cartilage lesions: from preclinical findings to clinical application in orthopaedics. KSSTA. 2013;21:1717-29.

16. Somoza RA, Welter JF, Correa D, Caplan Al. Chondrogenic differentiation of mesenchymal stem cells: challenges and unfulfilled expectations. Tissue Eng Part B Rev. 2014;20:596608.

17. Singer NG, Caplan AI. Mesenchymal stem cells: mechanisms of inflammation. Annu Rev Pathol. 2011;6:457-478.

18. Lodi $D$, lannitti T, Palmieri B. Stem cells in clinical practice: applications and warnings. J Exp Clin Cancer Res. 2011;30:9.

19. Guo W, Wang H, Zou S, Gu M, Watanabe M, Wei F, et al. Bone marrow stromal cells produce long-term pain relief in rat models of persistent pain. Stem Cells. 2011;29:1294-303.

20. Mcllwraith CW, Frisbie DD, Rodkey WG et al. Evaluation of intra-articular mesenchymal stem cells to augment healing of microfractured chondral defects. Arthroscopy. 2011;27: 1552-61.

21. Nam H, Karunanithi $P$, Loo W, Naveen $S$, Chen $H$, Hussin $P$, Chan L, Kamarul T. The effects of staged intra-articular injection of cultured autologous mesenchymal stromal cells on the repair of damaged cartilage: a pilot study in caprine model. Arthritis Res Ther. 2013,15:R129.

22. Saw KY, Hussin P, Loke SC, et al. Articular cartilage regeneration with autologous marrow aspirate and hyaluronic acid: an experimental study in a goat model. Arthroscopy. 2009; 25:1391-1400.

23. ter Huurne M, Schelbergen R, Blattes R, et al. Antiinflammatory and chondroprotective effects of intraarticular injection of adipose-derived stem cells in experimental osteoarthritis. Arthritis Rheum. 2012;64:3604-3613.

24. Jo CH, Lee YG, Shin WH, et al. Intra-articular injection of mesenchymal stem cells for the treatment of osteoarthritis of the knee: a proof-of-concept clinical trial. Stem Cells. 2014;32: 1254-66.

25. Koh YG, Choi YJ, Kwon SK, Kim YS, Yeo JE. Clinical results and second-look arthroscopic findings after treatment with adipose-derived stem cells for knee osteoarthritis. Knee Surg Sports Traumatol Arthrosc. 2015;23:1308-16.

26. Suh K-T, Kim S-W, Roh H-L, Youn M-S, Jung J-S. Decreased osteogenic differentiation of mesenchymal stem cells in alcohol-induced osteonecrosis. Clin Orthop Relat Res. 2005; 431:220-225.

27. Koh YG, Choi YJ. Infrapatellar fat pad-derived mesenchymal stem cell therapy for knee osteoarthritis. Knee. 2012; 19:902-907. 